\title{
Operation of an electron-cyclotron-resonance ion source with supplemental electron injection
}

\author{
I. Boscolo* and S. Cialdi \\ University and INFN, Via Celoria 16, 20133 Milano, Italy \\ S. Gammino and G. Ciavola \\ INFN-LNS, Via Sofia 44, I-95123 Catania, Italy \\ (Received 16 April 2003; published 17 July 2003)
}

\begin{abstract}
Experimental observations obtained on an electron-cyclotron-resonance ion source with electron injection by a ferroelectric cathode are presented. The results are in reasonable agreement with the proposed qualitative plasma model based on the Fokker-Planck equation. A procedure for the optimized operation of an ion source with electron injection is presented on the base of the experimental observations.
\end{abstract}

DOI: $10.1103 /$ PhysRevSTAB.6.073501

PACS numbers: 52.50.Gj, 52.65.Ff, 79.75.+g, 77.80.-e

\section{INTRODUCTION}

An electron-cyclotron-resonance ion source (ECRIS) has the feature of a high current of multiply charged ions (MCIs) when the following four conditions are met [1-3]: (1) an electron temperature $T_{e}$ high enough for the atomic inner electron stripping (in our source of the order of some $\mathrm{keV}$ ), (2) a good confinement of hot electrons, which means both a relatively high electron lifetime $\tau_{e}$ and a relatively high electron density $n_{e}$ [4], (3) an ion lifetime $\tau_{i}$ long enough for the multiply charged ion creation through a step-by-step stripping process, and (4) a neutral gas pressure so low $\left(p<4 \times 10^{-7}\right.$ mbar $)$ that the rates of the two processes of ion-ion exchange and electron-ion recombination are much lower than the rate of the electron stripping process.

The optimized operation of the source is obtained through a proper setting of the three parameters gas pressure $p$, microwave power $P_{\text {rf }}$, and confining magnetic field $B$. In the procedure of searching the highest MCIs current, the plasma electron density and temperature are driven towards the right values. At that point, the plasma has a stable configuration and a positive potential $V_{p}$ with respect to the plasma chamber [5]. This potential plays a relevant role in the ionization process, because it contributes in determining the ion/electron lifetime in the plasma.

Two models are proposed for ion confinement in ECRIS [6]: (1) the model of the potential dip (where electrons are trapped in a potential well set in the center of a plasma bubble), and (2) the model of ion diffusion within the electric field created inside the plasma. We will assume this latter model in the following (according to Refs. [5,6]). For the plasma dynamics we assume the model of Ref. . In this model the ions are cold and highly collisional; they can be assumed to have a Maxwellian

\footnotetext{
*Author to whom correspondence should be addressed.
}

Electronic address: ilario.boscolo@mi.infn.it distribution function. The average ion confinement time $\tau_{i}$ is determined by the plasma potential, which acts upon the ions and expels them from the plasma. The ECRIS stable plasma configuration is dynamical: the ion loss is compensated by an equivalent electron loss and the lost ions and electrons are refilled via the ionization of atoms entering the plasma. The ion current is

$$
J_{i}=q_{i} n_{i} \mu_{i} E-q_{i} D_{i} \frac{d n_{i}}{d x}, \quad \mu_{i}=\frac{q_{i}}{m_{i} \nu},
$$

where $J_{i}, \mu_{i}, q_{i}, D_{i}, n_{i}, m_{i}, \nu$, and $E$ are, respectively, the ion current density, the ion mobility, the ion charge, the diffusion coefficient, the ion density, the ion mass, the ion frequency collision, and the electric field. The plasma voltage $V_{p}$ depends on the many plasma parameters

$$
V_{p}=f\left(n_{e}, n_{i}, n_{n}, T_{e}, T_{i}, B\right)
$$

where $n_{n}$ and $T_{i}$ are the neutral density and the ion temperature, respectively. The plasma chamber configuration and wall condition affect the value of $V_{p}$ as well.

The implementation of an electron source in an ECRIS adds a new knob capable of controlling the value of $n_{e}$ through electron current injection. Electron injection most likely increases the plasma electron density and reduces the plasma potential. In the experiment of Ref. [5], it was measured that $V_{p}$ decreased and the ion current increased when the electron injection was switched on. We may infer that the plasma dynamical equilibrium is changed towards a higher electron current flowing out from the plasma, being this current is partially compensated by the injected electron current. A new parameter $i_{e x}$ must be added to the set of parameters bracketed on the left side of Eq. (2).

In this article we discuss the possible positive role played by electron injection into an ECR plasma in the fulfillment of the requested conditions of high $T_{e}$, high $\tau_{i}$, and low $p$. The application to ECRIS sources of systems capable of supplying electrons to the plasma for 


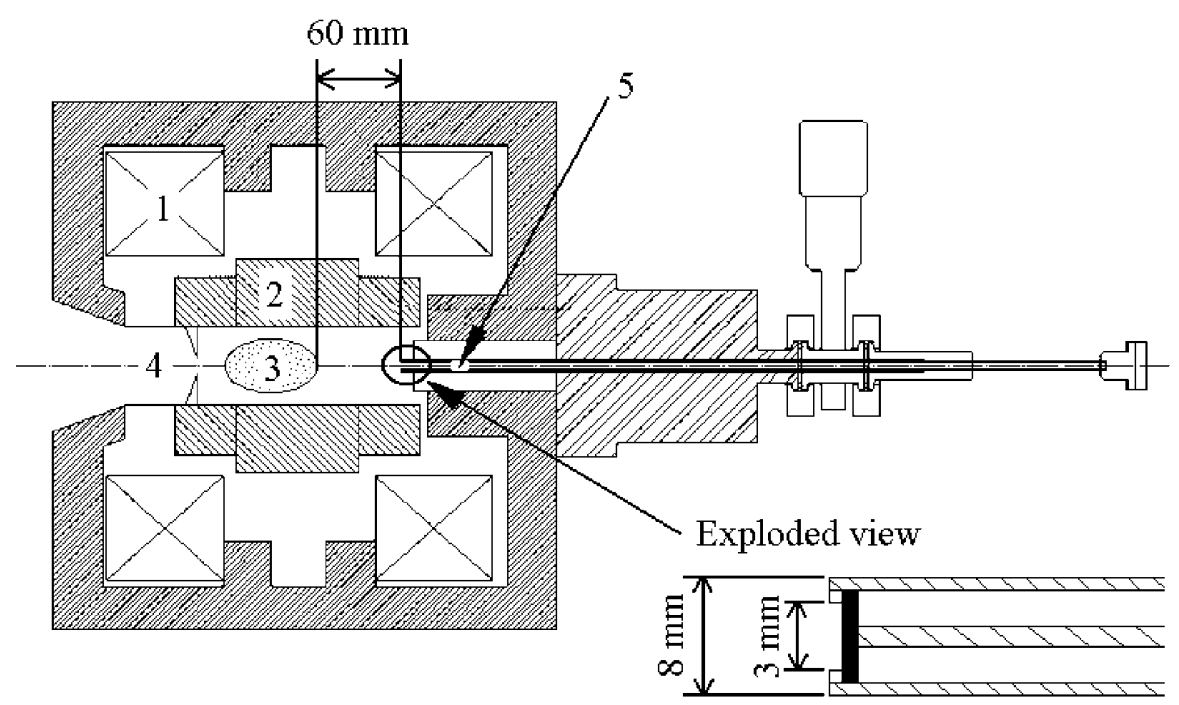

FIG. 1. Basic test setup with an ECR ion source chamber: (1) the coils, (2) the hexapole, (3) the ECR plasma surface, (4) the extractor, and (5) the ferroelectric cathode are shown.

compensating the electron escape from the loss cone was made successfully a few years ago [7]. Different systems had been used throughout the years, since, for the first time, the wall coating was perceived to be an efficient means of yield enhancement [8]. In the system with the wall coating the shape of the charge state, distribution did not change (i.e., the amount of the increase is the same for every charge state). The source improvement was explained in terms of an electron density growth because of the refilling of the electrons lost by the plasma; the amount of ionizing interactions is increased, thus making the plasma a much more efficient ionizing environment. Electron injection, by a dedicated electron gun, was also experimented with having good success [9]. In the early 1990's experiments in Grenoble [10] and at KVI Groningen have shown that the presence of a negatively biased metallic disk improves the ECR source performance $[11,12]$. This effect was explained by an increase of electron density affecting the output current intensity level. The plasma confinement is not modified, though the source stability is improved, and the optimization of the highest charge states is easier.

We are proposing again the technology of the electron gun because an electron gun based on the ferroelectric cathode seems tailored for this implementation. The electron gun is located on the axis at the injection side; see Fig. 1. Its longitudinal position is just after the peak of the confining magnetic field.

\section{THE FERROELECTRIC CATHODE FEATURES}

The general discussion on this cathode for the ECRIS machine and the experimental setup in the CAESAR ion source is presented in Ref. [13]. The ferroelectric cathode was inserted into the injection channel of the ion source as shown in Fig. 1. Here we stress both the capability of the cathode to operate at any environmental condition of temperature (less than $500{ }^{\circ} \mathrm{C}$ ) and pressure and the fact that bare ferroelectric disks emit electrons in the range of 0-2 keV without any external accelerating field (depending on the excitation [14]). Further, the disk diameter can be as small as a few millimeter (the current reduces in proportion); thus it can be tailored to any kind of source. In this connection, we remark that the cathodes must be small enough to avoid outgassing and cavity tuning problems. The cathode could be easily moved along the axis for tests at different distances from the plasma, therefore in different physical environments, being magnetic field, temperature, pressure, and electric field (because of the plasma voltage) different in each position. We believe that the magnetic field configuration is particularly important for an efficient driving of the emitted electrons into the plasma bubble.

The ferroelectric cathode implemented in the ion source for the tests provided pulses of a couple hundred nanoseconds for a charge per pulse of $1 \mathrm{nC}$. The frequency of operation was fixed at $250 \mathrm{~Hz}$. The cathode for tests reported in this article was set at $6 \mathrm{~cm}$ from the plasma border; see Fig. 1. The cathode material was lead zirconate titanate lanthanum doped ceramics (PLZT) of composition $4 / 95 / 5$, where the first number refers to the relative percentage of $\mathrm{La}$ with respect to $\mathrm{Pb}$ and the other two numbers refer to the ratio $\mathrm{Zr} / \mathrm{Ti}$ [15]. This material was chosen for its resistance to fatigue [14] and because it can operate at a relatively high repetition rate (few $\mathrm{kHz}$ ) with an acceptable amount of charge per pulse. We stress that a ferroelectric cathode can operate with different current intensity and with different electron energy depending on the excitation pulse characteristics, that is rise time and half or full square wave [14]. 
About the present experiment, we point out that the so far developed ferroelectric source was constrained into a too small space for reliable operation at the $2 \mathrm{kV}$ voltage amplitude required for the excitation (see exploded view in Fig. 1). We decided to do the tests without any substantial modification of the system for gaining ideas after the first results. The ferroelectric disk was simply implemented into the existing small tube (designed, at the time of the machine, for plasma diagnostic purposes) and the default driving high voltage pulser was used. We would say now that the results were satisfactory, but the challenge was too risky: the pulser broke down after hours of operation, maybe because of possible short-circuits. Unfortunately, it broke down during a run which was giving important scientific outcomes; see next section.

\section{THE SEARCH FOR THE OPTIMIZED SOURCE OPERATION}

The optimized set of plasma parameters is reached through a process of continuous and correlated increase of $B, P_{\mathrm{rf}}$, and a decrease of $p$. Now we discuss a possible change due to the electron current injection.

\section{A. The microwave power without and with electron injection}

The MCIs output current of an ECRIS depends on the set of the three parameters governing the plasma configuration, that is the confining magnetic field, the neutral pressure and the microwave power. It depends on the path followed by the operator to reach the source optimization as well.

First experiment: behavior of the source set at the optimized configuration at the activation of the electron gun. We did the tests at different values of the parameters. A typical set was $p_{\text {neutral }} \approx 2.5 \times 10^{-7} \mathrm{mbar}, P_{\mathrm{rf}} \approx$ $400 \mathrm{~W}$. We observed a reduction of the MCIs output current any time the electron source was activated. Figure 2 is a typical output: it shows a relevant decrease of the high MCIs species and a small increase of the lowest specie. This behavior leads one to conclude that the two possible negative effects, the increase of the recombination processes' rate (as a consequence of the cold electron density enhancement) and the decrease of the hot electron group, are effective.

We notice that the initial setting of the system parameters (hence also of the microwave power level) for the highest MCIs determines both the temperature and the population of the hot electrons. The injection of cold electrons into the plasma with that configuration leads to a temperature reduction (the electron population is increased at stable heating power). This view is in agreement with the results of the model of Ref. [1], in which it is shown that the maintenance of the electron temperature with the increase of the electron density requires a very high increase of microwave power. These considerations

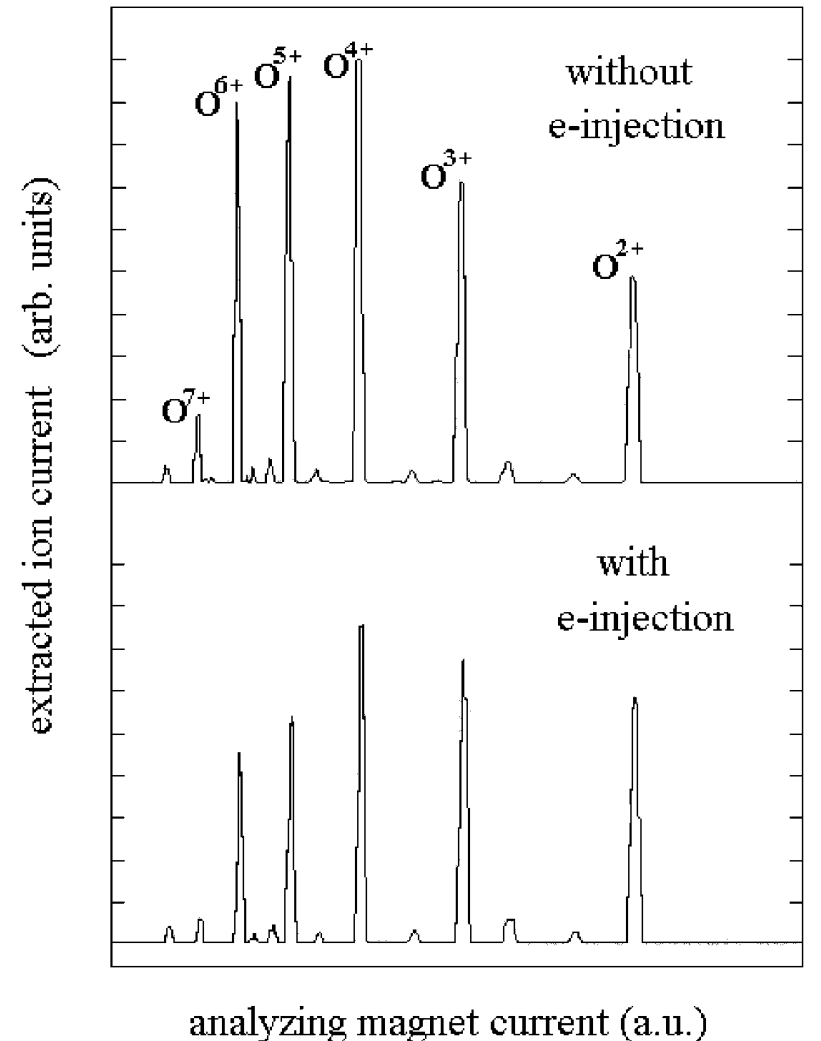

FIG. 2. The two frames show the charge state distribution before and after the electron injection: The reduction of the highly charged ions and the enhancement of the lowly charged ones is evident.

lead one to conclude that, when the electron injection is inserted in a machine, a higher $P_{\mathrm{rf}}$ than that required without electron injection must be set in the optimization procedure. This conclusion is completely supported by the result shown in Fig. 3.

Second experiment: behavior of the source at high microwave power. The system was set at $700 \mathrm{~W}$ microwave power, which is a high power level for our source, and then electron injection was switched on (the initial MCIs emission with the new set of parameters was a bit lower with respect to the emission at the optimized configuration). The plasma showed some instability. At the turning on of the injection current, the MCIs $\mathrm{O}^{7+}$ current started to increase linearly, as shown in Fig. 3, and the plasma became quiescent. Unfortunately, while the system was evolving towards higher and higher MCIs current, the ferroelectric electron source broke down abruptly and we could not fix it anymore (the cathode needs a technological improvement). The current increased more than $20 \%$, but, more important, a further consistent enhancement can be expected (no sign of saturation is present in the curve of Fig. 3). This is consistent with the picture of a more dense plasma and lower plasma voltage (with electron injection). The move of the plasma 


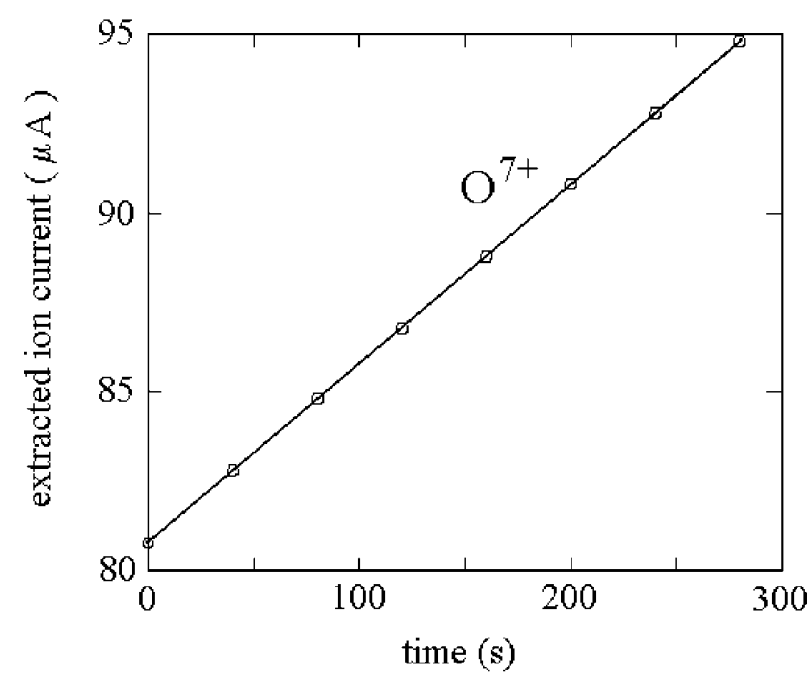

FIG. 3. The diagram shows the increase of the MCIs current with time after the switching on of the electron source. The stop of the curve was due to a destructive discharge at the ferroelectric cathode.

from an unstable to a stable configuration was observed also in experiments reported in Refs. [5,13].

\section{B. The neutral pressure without and with electron injection}

The electron injection increases the plasma electron density $n_{e}$. Since MCIs production requires large electron energy to overcome the ionization potential and high electron energy requires low pressure [2,6], external electron injection must be applied with a coherent reduction of neutral injection. In our positive test the pressure was, in fact, reduced by about a factor of 2 , passing from the $2.7 \times 10^{-7}$ mbar of the first experiment to the $1.35 \times$ $10^{-7}$ mbar of the second experiment.

We notice that the compensation of electron losses by external electron injection enables the maintenance of a low density of neutral. This low density means a small rate of the charge exchange process between ions and atoms, with a beneficial effect on the MCIs production. In addition, the reduction of neutral density (gas flow) means a reduction of ion density, hence a reduction of the ion diffusion coefficients.

The link between the electron injection and the neutral flux indicates that, in the optimization procedure, the presence of the electron injection asks for a consequent reduction of the neutral flux.

\section{The plasma potential without and with electron injection}

In the experiments of Refs. [5,16], it was measured that electron injection into an ECR plasma leads to a reduction of the plasma potential $V_{p}$. This result is seemingly due to the compensation of electron losses by the injected electrons [17]. We notice that the losses are relatively high at the high working $P_{\mathrm{rf}}$ requested by the efficient MCIs production.

The measured enhancement of the MCIs current with electron injection supports the model of ion transport in the ambipolar electric field [6]. In fact, the model of the ion trapping in a potential dip $[16,18,19]$ supposes a negative potential well produced in the center of the plasma bubble by high-energy electrons. The injected cold electrons seemingly operate to reduce the temperature of the hot electron group because of collisions. However, there are two countereffects with electron injection: one is the presence of a higher microwave power and the other is the reduction of the neutral gas pressure. The higher microwave power into the plasma enhances its temperature and the lower neutral pressure leads to a longer lifetime of the hot electron group. The observed reduction of the plasma potential indicates that the combination of the cited effects reduces the density of the hot electrons but increases the ion lifetime at such an extent that the rate of the highly ionized ion production increases. Therefore, the reduction of the positive $V_{p}$ is more likely the most important reason of the MCIs current enhancement.

We would conjecture that the hot electron group is not affected by the arrival of cold electrons into the plasma. This is a demonstration of the validity of the transport model for our plasma. Reference [2] states that the $\tau_{e}$ is inversely proportional to the neutral pressure and that the neutral pressure has to be kept low in order to get high $\tau_{e}$ which in turn leads to high $\tau_{i}$ and $T_{e}$. In fact, the ion lifetime is closely related to $\tau_{e}$ because of quasineutrality and $T_{e}$ increases as well if a sufficient power is provided.

\section{THE PLASMA RESONANT SURFACE POSITION WITHOUT AND WITH ELECTRON INJECTION}

The magnetohydrodynamic condition for plasma confinement $\nabla \times\left(p_{\perp}+B^{2} / 2 \mu_{0}\right)=0$ is only partially satisfied in ECRIS systems. These systems are open systems because ions are programmed to leave the plasma and consequently the plasma equilibrium is dynamical. There are two about equal output currents of electrons and ions. In ideal ECRIS systems the maximum magnetic field $B_{\max }$ and the magnetic field at the resonance surface $B_{\mathrm{ECR}}\left[B_{\mathrm{ECR}}=(m / e) \omega_{\mathrm{rf}}^{2}\right]$ must fulfill the following condition:

$$
\frac{B}{B_{\mathrm{ECR}}}>2
$$

in order to get the optimum plasma confinement [4,17]. This condition, called high $B$ mode of operation, was proposed in 1990 and tested by many experiments $[10,12]$. When the system has a current injection, the continuity equation has an extra term $\nabla \vec{J}_{\text {ext }}$ due to this 
inlet current. The stability condition (3) for the plasma, when the electron losses are compensated by the external injected electrons, should relax towards a lower value of the maximum magnetic field. The resonant surface can be moved outward. This magnetic field configuration allows a wider ECR resonant zone (with an increase of the microwave efficiency absorption) and, in turn, the generation of the hot electrons in a more external surface of the plasma bubble. The closeness of the ions to the capturing zone of the ion gun reduces the recombination and ion-ion exchange probability [20,21]. The observed relaxation of the plasma towards quiescence, previously presented, could be explained in the framework of the enhanced stability by the action of the injected current.

The magnetic field level at the activation of the ferroelectric electron source has to be set at a lower level than the level required without the electron source. In fact, the presence of a high electron density on axis at the injection side provides an additional hurdle to the electron migration outward, so that a lower magnetic field is sufficient to trap the electrons which are slowed down or reflected from the electric field generated by the space charge. As the electron density is increased at the opposite side with respect to the extraction system, a preferred direction for electron migration is set by the space charge generated by the electrons. This field configuration favors the enhancement of ion extraction, which is generally obtained by means of a strongly asymmetric magnetic field.

\section{CONCLUSIONS}

The implementation of a ferroelectric electron source in an ECR ion source is easy and convenient for multiply charged ion enhancement. The tests have shown that a pulsed electron source (as one provided by a ferroelectric cathode) can be fruitfully used. The first experimental observations indicate that an ECRIS with an external electron injection has a set of machine parameters for optimized operation different from those of a source without the electron injection: the microwave power is higher, while the neutral pressure and the confining magnetic field are lower. The behavior of the source suggests the following possible picture for the dynamical plasma equilibrium: electrons provided to the plasma compensate for electron losses. Therefore, the equilibrium values of the electron and ion densities move towards a lower difference between the two species, reducing the plasma voltage and increasing the ion lifetime (and, consequently, their charge state). The higher microwave power level accepted by that plasma, in proportion to the neutral decrease, indicates that the electron temperature increased and highly charged ions is increased too. The rate of recombination processes is reduced. A better plasma quiescence is observed.

The ferroelectric cathode is tailored for the implementation of an electron source inside an ECRIS ion machine for its capability to operate within a plasma ambient and for its easy handling. The possibility to operate at different current intensities and electron energies is another interesting feature. Our first two series of tests of the ferroelectric cathodes have shown that the cathodes must be improved from the technological point of view so to resist the couple of kilovolts required by excitation even dealing with disks whose dimensions are less than $10 \mathrm{~mm}$ diameter. Some technological modifications are under way. We believe that systematic tests as a function of the many variables (excitation pulse amplitude, pulse shape, repetition rate, distance from the plasma) must be done in order to find out the best system configuration.

\section{ACKNOWLEDGMENTS}

The support of F.G. Caruso, M. Castro, F. Chines, E. Messina, S. Marletta, and E. Robert (LNS) is gratefully acknowledged. The contribution of D. Cipriani (Milan) in growing the ceramics and preparing the cathodes is recognized.

[1] A. Girard, C. Pernot, G. Melin, and C. Lécot, Phys. Rev. E 62, 1182 (2000).

[2] C. Perret, A. Girard, H. Khodja, and G. Melin, Phys. Plasmas 6, 3408 (1999).

[3] A. Girard, C. Perret, G. Melin, and C. Lécot, Rev. Sci. Instrum. 69, 1100 (1998).

[4] S. Gammino and G. Ciavola, Plasma Sources Sci. Technol. 5, 19 (1996).

[5] Z. Q. Xie and C. M. Lyneis, Rev. Sci. Instrum. 65, 2947 (1994).

[6] G. Douysset, H. Khodja, A. Girard, and J. P. Briand, Phys. Rev. E 61, 3015 (2000).

[7] S. Gammino and G. Ciavola, in High Charge State ECR Ion Sources: Status and Developments, Proceedings of the 8th Conference on Heavy Ion Accelerator Technology, edited by K. Shepard, AIP Conf. Proc. No. 473 (AIP, New York, 1999), p. 300, and references therein.

[8] R. Geller, Electron Cyclotron Resonance Ion Sources and ECR Plasmas (Institute of Physics Publishing, Bristol, 1996).

[9] C. Lyneis and Z.Q. Xie, in Proceedings of the 10th International Workshop on ECR Ion Source, Oak Ridge, TN, 1990, edited by F.W. Meyer and M. I. Kirkpatrick (Oak Ridge National Laboratory, Oak Ridge, TN, 1991), p. 47.

[10] R. Geller, in Proceedings of the 10th International Workshop on ECR Ion Source, Oak Ridge, TN, 1990 (Ref. [9]), p. 381.

[11] S. Gammino, J. Sijbring, and A. G. Drentje, Rev. Sci. Instrum. 63, 2872 (1992).

[12] G. Ciavola, S Gammino, T. Antaya, and K. Harrison, in Proceedings of the 12th International Workshop on ECR Ion Sources, RIKEN, Tokyo, 1995, edited by 
M. Sekiguchi and T. Nakagawa (Institute for Nuclear Study University of Tokyo, Tokyo, 1995), p. 156.

[13] I. Boscolo, S. Cialdi, M. Valentini, S. Gammino, G. Ciavola, L. Celona, S. Marletta, H. Riege, and J. Handerek, J. Appl. Phys. 90, 2447 (2001).

[14] I. Boscolo and S. Cialdi, J. Appl. Phys. 90, 6341 (2001).

[15] G. Benedek, I. Boscolo, J. Handerek, and H. Riege, J. Appl. Phys. 81, 1396 (1997).

[16] G. Melin, F. Bourg, P. Briand, J. Debernardi, M. Delaunay, R. Geller, B. Jacquot, P. Ludwig, T. K.
N'Guyen, L. Pin, M. Pontonnier, J.C. Rocco, and F. Zadworny, Rev. Sci. Instrum. 61, 236 (1990).

[17] S. Gammino and G. Ciavola, Rev. Sci. Instrum. 71, 631 (2000).

[18] D. Bolshukhin et al., Rev. Sci. Instrum. 69, 1197 (1998).

[19] T. Nakagawa, Rev. Sci. Instrum. 69, 637 (1998).

[20] G. D. Alton and D. N. Smithe, Rev. Sci. Instrum. 65, 775 (1994).

[21] Y. Liu, G. D. Alton, G. D. Mills, C. A. Reed, and D. L. Haynes, Rev. Sci. Instrum. 69, 1311 (1998). 